\title{
Safe care on maternity units: a multidimensional balancing act
}

\author{
Emily White VanGompel, ${ }^{1}$ Elliott K Main ${ }^{2}$
}

${ }^{1}$ Departments of Family Medicine and Obstetrics and Gynecology, University of Chicago Pritzker School of Medicine, Evanston, IL, USA ${ }^{2}$ Obstetrics and Gynecology / California Maternal Quality Care Collaborative, Stanford University School of Medicine, Palo Alto, CA, USA

\section{Correspondence to}

Dr Elliott K Main, Pediatrics, Stanford University School of Medicine, Palo Alto, CA 94305, USA; emain@stanford.edu

Accepted 5 January 2021 Published Online First

15 January 2021

\section{Sinked}

- http://dx.doi.org/10.1136/ bmjqs-2020-010988

\section{Check for updates}

(c) Author(s) (or their employer(s)) 2021. No commercial re-use. See rights and permissions. Published by BMJ.

To cite: White VanGompel E, Main EK. BMJ Qual Saf 2021:30:437-439.
What are the key features of hospitals that consistently deliver safe care on labour and delivery? This is the primary question posed by Liberati and colleagues in this issue of BMJ Quality \& Safety. ${ }^{1}$ The authors propose a framework distilled from observations on a group of highperforming units in the UK participating in a training activity to improve patient safety. This study combined ethnography with individual interviews and focus groups and involved over 400 hours of total observations at six different maternity care sites. The seven features in their resulting For Us framework correspond well to existing theoretical as well as applied quality improvement strategies. While we agree that their framework describes features that every labour and delivery unit should strive to include, this approach has some limitations in terms of generalisability. Specifically, Liberati and colleagues studied maternity units that are high performing, but their sample included only large-volume hospitals in what appear to be well-resourced settings. What is potentially missing is observations on underperforming units, and how these findings may or may not apply to smaller, lower resourced settings. Additionally, the structure of the UK's National Health Service (NHS) also limits generalisability. For example, this is most analogous to employed physician models in the USA, with the potential advantage of a more organisationally oriented provider workforce. Given that most US hospitals do not have an employed provider model, we can't assume that these factors will have the same impact in other models of care.

In the USA, the Agency for Healthcare Research and Quality (AHRQ) developed a Culture of Safety framework that delineates four key features: (1) organisations recognise that their primary activities are inherently high risk and make it their goal to operate in a reliably safe manner; (2) organisations create a safe and blame-free reporting environment; (3) interdisciplinary and interprofessional collaboration is encouraged to address safety problems; and (4) resources are deliberately allocated and made available to address safety. ${ }^{2}$ This framework, as does For Us, focuses on a healthcareoriented conceptualisation of safety and quality, and details medical outcomes as the primary metrics by which to measure success. Although achievement of these medical quality outcomes is imperative, we propose that there are additional domains needed to provide safe intrapartum care: (A) prioritising patient experience-including emotional safety, birthing with dignity and an expectation of person-centred care; and (B) a unit culture that values low intervention births. Let us consider these domains in more depth.

Patient experience and safety are inextricable. While much work has been done to improve physician-patient communication, ${ }^{34}$ few have successfully targeted the perpetuation of dysfunctional behaviours grounded in healthcare professionals' implicit and explicit biases. ${ }^{5}$ This may be in part due to the tendency to observe and look for answers from the standpoint of the healthcare system rather than patients. Women who had recently given birth were included in the study of Liberati and colleagues, but represented only 8 of 65 individual stakeholder interviews, and were not included in focus groups. The framework thus describes a high-functioning system from primarily the healthcare system's perspective. In general, the patient's role in achieving safe care includes many aspects, including providing personal information to reach the correct diagnosis, providing their values and lived experience in shared decision-making discussions, choosing 
their provider such that their needs regarding provider experience and safe practice are met, making sure that they receive the recommended treatments in a timely manner, as well as identifying and reporting errors. ${ }^{6}$ The detriment to health outcomes among patients who have failed interactions with providers is well documented (eg, leaving against medical advice or experiencing disrespect during their care) while other harms, such as psychological trauma, often go unmeasured. ${ }^{7}$

Emotional and psychological trauma are safety errors, whether or not a patient leaves the hospital physically intact. ${ }^{8}$ Research has shown that patients experience psychological trauma both as a result of an adverse outcome and as a result of how the incident was managed. In birth, patients conceptualise the meaning of safety very differently from that of the medical system, with physical and emotional safety being inextricably interwoven into a single concept. ${ }^{9}$ Psychological trauma may manifest in postpartum depression, post-traumatic stress disorder ${ }^{10}$ and, some studies suggest, reduced childbearing in patients who experience traumatic birth. ${ }^{11}$ The experience of emotional safety on the part of the patient is only knowable to the patient, and only addressable when health systems - and health services research—ask the appropriate questions. Therefore, patient-reported experience measures and critical examination of the process of patient-centred care should be at the centre of quality improvement.

High-performing units prioritise patient voice and patient experience as a part of their culture. In a recent article, Morton and Simkin ${ }^{12}$ delineate steps to promote respectful maternity care in institutions, including obtaining unit commitment to respectful care, implementing training programmes to support respectful care as the norm and, finally, instituting respectful treatment of healthcare staff and clinicians by administrators and leaders-in other words, a unit culture of mutual respect and care among the entire team enables respectful care of the patient. Liberati and colleagues address the issue of hierarchies on labour and delivery, making the key observation that high-performing units create hierarchies around expertise rather than formal titles or disciplinary silos; however, this power differential applies to patients as well. The existing hierarchy on most labour units places physicians at the top and patients at the bottom, which often acts to silence patients' voices. ${ }^{13}$ Implicit bias and interpersonal racism and sexism contribute to this cycle of silence and mistreatment on labour and delivery units. ${ }^{14}$ Disrespect and dismissal of patient concerns have been increasingly described, but still lack quantitative measurement in association with maternal and child health outcomes. ${ }^{15}$ Interventions aimed at harm reduction are emerging, ${ }^{16}$ but more work is desperately needed in this area.

Valuing low intervention is an important dimension of safety. Safety culture, as it is conceptualised by AHRQ and the current study, is ideally created to prevent or respond to harmful safety lapses. This model is more difficult to apply to an environment where the goal is safe facilitation of a normal biological process. In this setting, interventions (that often beget more interventions) can increase complications. High rates of primary and repeat caesarean deliveries, and other invasive obstetric interventions seen in many birthing units are now widely acknowledged to be overused and overuse constitutes a patient safety risk. ${ }^{17}$ In our work in California, we have been able to demonstrate that provider attitudes, beliefs and unit culture can drive caesarean delivery overuse in ways that do not contribute to patient safety. ${ }^{18} 19$ Each intervention needs to be carefully and jointly considered for value and safety. This in no way diminishes the life-saving nature of caesarean delivery when it is medically indicated, but it sets up the expectation that safety measures, processes and procedures must be in place to actively work towards supporting vaginal birth rather than treating each labour as an emergency waiting to happen. The striking variation in obstetric intervention rates among hospitals and providers can provide critical insights. So, what is the right balance of intervention rates and mother/baby safety outcomes? In many instances, this may be a false dichotomy. In a study of California hospital labour practices, Lundsberg et al found that hospitals that prioritised low labour interventions and actively supported vaginal birth (eg, delaying admission until active labour onset, use of doulas, intermittent auscultation of fetal heart tones, non-pharmacological pain relief, and so on) had reduced caesarean delivery rates with well-preserved neonatal outcomes. ${ }^{20}$ It should be noted that in the USA, rates of intervention are starting at a high level so there is less danger of harm from achieving too low a rate. This may not be the case in the UK where there are now formal inquiries examining obstetric care in multiple NHS hospital trusts where poor perinatal outcomes have been linked to a systematic aversion to medical interventions even when indicated. ${ }^{21}$ Getting this balance right has been referred to as the Goldilocks quandary: Doing too little, too much or just right? ${ }^{22}$

In conclusion, physical safety is the bare minimum of what should be expected in childbirth. Patients have a right, and healthcare providers and systems have an obligation to aim higher, to ensure patients emerge from childbirth as healthy or healthier-both physically and psychologically-than before entering the hospital. This can be best achieved by broadening the lens of what we consider essential to safety on maternity units to include prioritising patient experience, birthing with dignity and valuing low intervention rates. All of these domains need to be in balance: good mother or baby medical outcomes at the cost of high rates of intervention and high maternal psychological trauma are not a success, nor is the opposite. The true 'safe' maternity unit is one that does well on all 
of these dimensions, which, of course, means that we need to be able to measure each of them. Finally, all of these safety domains, including the 'For Us' framework proposed by Liberati and colleagues, focus on unit culture, provider behaviours and processes of care, and thus are within the reach of all maternity units no matter their level of resources.

Funding The authors have not declared a specific grant for this research from any funding agency in the public, commercial or not-for-profit sectors.

Competing interests None declared.

Patient consent for publication Not required.

Provenance and peer review Commissioned; internally peer reviewed.

\section{REFERENCES}

1 Liberati EG, Tarrant C, Willars J, et al. Seven features of safety in maternity units: a framework based on multisite ethnography and stakeholder consultation. BMJ Qual Saf 2021;30:444-56.

2 Jones KJ, Skinner A, Xu L. The AHRQ Hospital Survey on Patient Safety Culture: A Tool to Plan and Evaluate Patient Safety Programs. In: Henriksen K, Battles JB, Keyes MA, eds. Advances in patient safety: new directions and alternative approaches (vol. 2: culture and redesign. Rockville (MD): Agency for Healthcare Research and Quality (US), 2008.

3 Detmar SB, Muller MJ, Schornagel JH, et al. HealthRelated quality-of-life assessments and patient-physician communication: a randomized controlled trial. JAMA 2002;288:3027-34.

4 Stewart MA. Effective physician-patient communication and health outcomes: a review. CMAJ 1995;152:1423-33.

5 FitzGerald C, Martin A, Berner D, et al. Interventions designed to reduce implicit prejudices and implicit stereotypes in real world contexts: a systematic review. BMC Psychol 2019;7:29.

6 Vincent CA, Coulter A. Patient safety: what about the patient? Qual Saf Health Care 2002;11:76-80.

7 Tucker Edmonds B, Ahlberg C, McPherson K, et al. Predictors and adverse pregnancy outcomes associated with antepartum discharge against medical advice. Matern Child Health J 2014;18:640-7.

8 Sokol-Hessner L, Folcarelli PH, Sands KEF. Emotional harm from disrespect: the neglected preventable harm. BMJ Qual Saf $2015 ; 24: 550-3$.
9 Lyndon A, Malana J, Hedli LC, et al. Thematic analysis of women's perspectives on the meaning of safety during hospitalbased birth. J Obstet Gynecol Neonatal Nurs 2018;47:324-32.

10 Ayers S, Bond R, Bertullies S, et al. The aetiology of posttraumatic stress following childbirth: a meta-analysis and theoretical framework. Psychol Med 2016;46:1121-34.

11 Preis H, Tovim S, Mor P, et al. Fertility intentions and the way they change following birth- a prospective longitudinal study. BMC Pregnancy Childbirth 2020;20:1-11.

12 Morton $\mathrm{CH}$, Simkin P. Can respectful maternity care save and improve lives? Birth 2019;46:391-5.

13 Lyndon A. Failure to rescue, communication, and safety culture. Clin Obstet Gynecol 2019;62:507-17.

14 Altman MR, Oseguera T, McLemore MR, et al. Information and power: women of color's experiences interacting with health care providers in pregnancy and birth. Soc Sci Med 2019;238:112491.

15 Sigurdson K, Morton C, Mitchell B, et al. Disparities in NICU quality of care: a qualitative study of family and clinician accounts. J Perinatol 2018;38:600-7.

16 Chidi E, Cahill EP. Protecting your birth: a guide for black mothers. New York times, 2020. Available: https:// www.nytimes.com/article/black-mothers-birth.html? searchResultPosition $=1$ [Accessed 18 Dec 2020].

17 Shaw D, Guise J-M, Shah N, et al. Drivers of maternity care in high-income countries: can health systems support womancentred care? Lancet 2016;388:2282-95.

18 White VanGompel E, Perez S, Datta A, Main EK, et al. Cesarean overuse and the culture of care. Health Serv Res 2019;54:417-24.

19 White VanGompel E, Main EK, Tancredi D, et al. Do provider birth attitudes influence cesarean delivery rate: a crosssectional study. BMC Pregnancy Childbirth 2018;18:184-92.

20 Lundsberg LS, Main EK, Lee HC, et al. Low-interventional approaches to intrapartum care: hospital variation in practice and associated factors. J Midwifery Womens Health 2020;65:33-44.

21 The Guardian. The observer view on the inquiry into maternity care at Shrewsbury and Telford Hospital NHS trust. Available: https://www.theguardian.com/commentisfree/2020/ dec/13/the-observer-view-on-the-inquiry-into-maternity-careat-shrewsbury-and-telford-hospital-nhs-trust [Accessed 18 Dec 2020].

22 Morgan DM, Main E, Gee RE. The Goldilocks quandary of health care resources: too little, too much, or just right? Obstet Gynecol 2016;127:1039-44. 